\title{
Smart Trash Bin
}

\section{Prathikshith Jain, Sowmya K, Ganesh Prasad Navada V}

Department of Computer Science, Srinivas Institute of Technology Valachil, Mangaluru, Karnataka, India

\begin{abstract}
Article Info

Volume 7, Issue 4

Page Number: 170-175

Publication Issue :

July-August-2021

\section{Article History}

Accepted : 06 July 2021

Published : 13 July 2021

The quantity of garbage in the city of Mangalore is increasing daily. The garbage needs to be gathered from several points of location and then need to be moved to garbage collection sites. These enormous number of garbage collection points itself seed pollution and also absence of waste management resources available by the government will have the severe impact on environment and surrounding community. In view of all these impacts on the community and environment the authors have lifted this issue to build an internet of things based smart trash bin in and around the city. These smart trash bins are very fruitful and time-efficient waste management system that will be effective in Mangalore's smart city project.

Keywords: Localization, Range free, Survey, Wireless sensor network, Mobile anchor, Vehicles
\end{abstract}

\section{INTRODUCTION}

The waste management system of our city needs a notable upgrade. Our quest is to make the trash disposal system of our emerging smart city, smart. The smart trash bin will have different varieties of bin like compartmentalised bins or single door bins but all are connected to the internet. An website will be able to track the location and quantity of trash in each bin. This information can then be served to people in need of a trash bin. The status of the bin can be tracked by the city municipal service. If the bins in a specific area are full, then the city can alert garbage collection services. Poor management can cause hygiene problems and problems that ruin the city's landscape, so regular management is necessary.
This project includes the design and development of waste management system intended for municipal areas. One of the classical city problems, is the habit of its people who do not care about cleanliness of their environment. Creating awareness on how to discard and manage garbage in a good manner has still not become an important thought. The government does not have an effective and efficient waste management system. Waste management system based on IoT technology is capable of handling waste management problems of the city efficiently. In this project, a prototype of an IoT based waste management system has been proposed, especially for solid waste, focusing on segregation and garbage collection phase. The system consists of three main subsystems: the hardware subsystem consisting of a trash bin equipped with sensors and 
communication array for monitoring the garbage status, controlling the garbage collection schedule and to send the usage statistics to the data collection subsystems. The other subsystems are data management and data visualization subsystem for management and analysis purposes to empty the bin.

\section{LITERATURE}

Smart Trash Bins have been researched since the 1990s. The most current related work is done by Zavare and his colleagues[1] on sensor nodes connected to an Arduino board based control station, that uses a GSM module to send the sensor nodes data by SMS to the garbage collecting vehicle and to a server hosting web application by a Wi-Fi connection. The sensor nodes of the smart bins rely on the ultrasonic sensor to sense the fullness percentage according to pre-calculated bin depth. Moreover, a GPS module is used to get the bin location. The GPS module and the ultrasonic sensor are controlled by Amica R2 NodeMCU microcontroller board which has a built-in Wi-Fi module, that is used to connect to the control station.

Another work on wireless sensor network is done by Singh, Mahajan and Bagai[2]. The bins in his work are equipped with an accelerometer sensor to sense the opening and closing of the bin lid, a temperature and humidity sensor to check the present organic waste, and an ultrasonic sensor to sense the fullness status of the bin. All these sensors are controlled by Zigbee Pro microcontroller board, which has a builtin Wi-Fi module that is used to send the sensors data to a gateway. This paper also used the same type of microcontroller board in the gateway to receive the bins data and send it to a control station, that contains a server, over GPRS. The server in the control station relies on Caspio database management system with a web based user interface.
A paper by Navghane, Killedar and Rohokale[3] examined the use of weight sensor and three IR sensors to check the fullness status of the smart bin and send the sensors data to a web page over Wi-Fi network to a mobile phone. The microcontroller board used in this paper was ARM LPC2148.

\section{Microcontroller}

The microcontroller is a processor that is used specifically for the sake of control. The microcontroller has a smaller form than computers in general, but the microcontroller is built with the same elements.

\section{Ultrasonic distance sensor (HC-SR04)}

Ultrasonic wave-based distance measuring sensor. The working principle of this controller is similar to ultrasonic radar. Ultrasonic waves are emitted by a trigger then received back by the ultrasonic echo. The distance between transmit time and receiving time is a representation of the distance of the object. Ultrasonic wave-based distance measuring sensor can be seen in the Fig.1

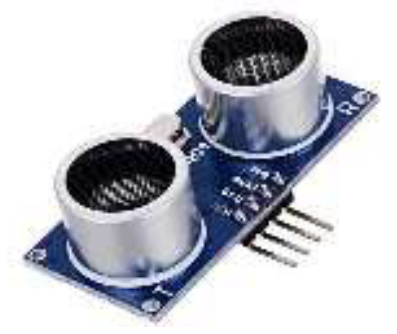

Fig. 1. Ultrasonic Distance Sensor (HC-SR04).

D. NodeMcu V3 Lua WIFI Internet of Things Development Board Based ESP8266.

The microcontroller development board was based on ESP8266 chip with a small form. This device was said as more practical and efficient because this thing was a microcontroller device that had been integrated with the wireless module without the need to add support devices to be able to connect to the wireless network where the prices offered were relatively cheaper, the device could be seen in the Fig.2 


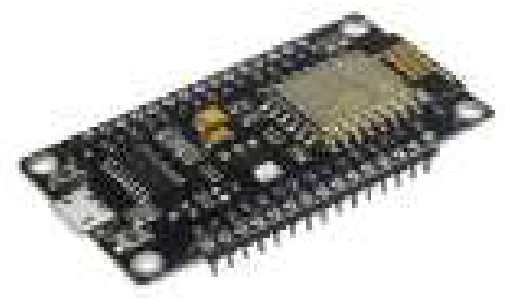

Fig. 2. NodeMcu V3.

\section{METHODOLOGY}

Data collection by making direct monitoring various objects, and the location of the existence of smart trash bins in the city of Mangalore. The process of dispatching information was done by spreading the points of the host to these locations, then through the internet network can monitor all the scattered waste bins, the results of monitoring as a decision to early detect the volume of garbage bins for transportation by trash transportation. The observation results will be used as a reference for making this research.

Research conducted in India, one of the developing countries in Asia [4], the proposed system used ultrasonic sensors to detect the quantity of waste. The tools used are Proximity Sensor, Arduino UNO, microcontroller and Raspberry Pi2.

Tool co-operation through the system globally shows that drivers of garbage collection vehicles would receive information via Message to the cell phone while the government could monitor the status of trash bins through web pages as well. One more study was still one country [4] that state waste production had increased more and need to implement intelligent systems in monitoring and disposal of waste. With the IoT-based approach (Internet of Things) researchers used ultrasonic sensors and MQ4, ultrasonic sensors to check the fullness of waste. The results of the study were referred to as "smart trash bin" which automatically separated recyclable trash from junk that could not be degraded biologically. If the garbage crosses the limit in a smart trash bin with junk that could not be degraded, the help of application information would be sent to the municipal officers for garbage disposal. If the junk crossed the limit of a smart trash bin with recyclable rubbish, the rubbish lid will slip and the junk would be disposed of into the underlying space. Research conducted in India (Asian Country) [5], that has the highest space of the country resulted in landfill waste in the surrounding environment, so an effective waste management system based on the IOT is needed. Systems with microcontroller areas an interface and the sensor system will detect the quantity of waste and then displayed it via GSM/GPRS based on Android.

Research conducted [6], that in some big cities in India(Bangalore), overflowing trash bins caused foul smell that would pollute the surrounding environment which results in the growth of bacteria's and viruses that results in various types of diseases such as Dengue. To overcome particular situation, an effective and efficient waste collection system was developed based on IoT.

The development of research in applying the Internet of Things (IoT) is carried out [7], Operation of a smart trash bin where the GSM board dispatches a message by finding the level of waste with the help of IR (Infrared Ray) installed in the trash bins. The main use of this system is how to control trash, as a human effort to realize smart cities.

The development of IoT was carried out in the India [8]. This study aimed to describe the security of the alarm system using a low processing power chip. IoT helped in knowing and getting alarms when a motion was detected and sending photos and videos to the cloud server. Also, Internet-based applications can be used remotely to see activities and get notifications 
when moment is detected. Pics and videos were dispatched directly to the cloud server when the cloud was not available then the data was stored locally in Raspberry Pi and would be sent if there was a connection. Hope this IoT application could monitor activity in a location.

Other related research [9] suggested that trash collection trucks in public places do not come regularly, resulting in more environmental pollution. Using the Less Energy Energetic Adaptive Hierarchy Algorithm (LEACH), the researchers proposed a less distance of the collection truck's travel time to the location of a garbage bin that was almost full and even detected the location of the trash that produced poisonous gas. The project was implemented and simulated using Network Simulator version 2 and the Arduino UNO developer board.

Research began to determine the data needed, and the tools needed. The starting stage of making a tool begins with the design of a microcontroller based on the ESP8266 chip to get signals from the ultrasonic distance sensor (HCSR04). The identification of early detection of trash cans built by the ultrasonic sensor HC-SR04 which will emit ultrasonic waves to an object, in particular case, was the location of the smart trash bins and then received the reflected waves, so that based on travel time (the starting time sending waves to receive back waves of reflection) could be known the distance between the sensors and the object. Then the information obtained was sent to the ESP8266 chip and processing the data. The ESP8266 chip would send information in the form of the volume of trash bins, through a wireless access point at the $2.4 \mathrm{GHz}$ frequency which would be forwarded to the server. The information would be on the cloud that would display on the website so that it could be easy to gain $70 \%$ waste bin information.

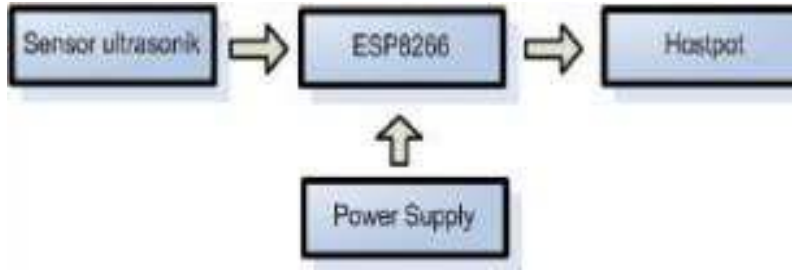

Fig. 3. System architecture

The client SSID put together a volume of garbage that had reached $70 \%$, then through the Wi-Fi signal the information would be forwarded to the webserver and then webserver would display early warning information about the trash.

\section{RESEARCH RESULT}

The outcome of this research project is verification of the development of smart trash bins by using information technology in smart cities where devices are built with consideration of less cost estimates and efficient device selection from previous studies. The proposed system is built for processing waste that is currently still being done manually, where the system can reduce the consumption of fuel from garbage collection vehicles. The mechanism for caution or sending signals produced by this smart trash bin was to read the distance of objects in front of the proximity sensor. The outcome of the distance reading of objects received by ultrasonic distance sensors that were sent to the microcontroller device which would be processed with decision-support algorithm formulas in smart bins and at the end of the process will be issued in the form of a percentage of the trash capacity conveyed to the webserver through the facility Hotspot.

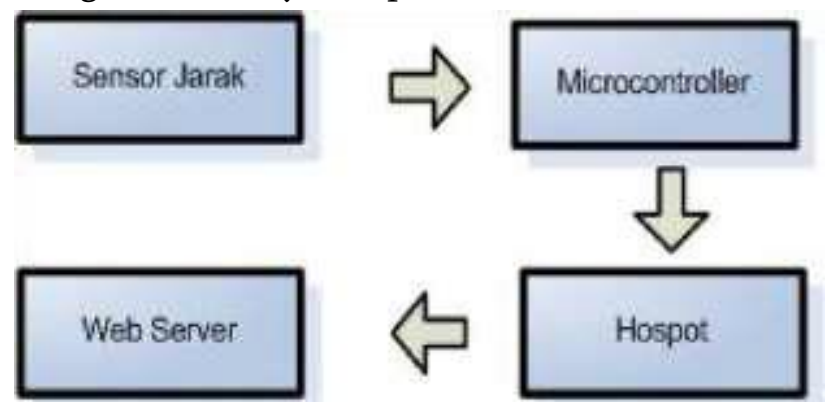

Fig. 4. Block Diagram of Device Prototype on Smart Trash. 
The design of the microcontroller system uses the basis of the ESP8266 module device embedded in the NodeMcu V3 and then coupled with input in the form of a proximity sensor to produce a warning signal dispatched directly to the webserver.

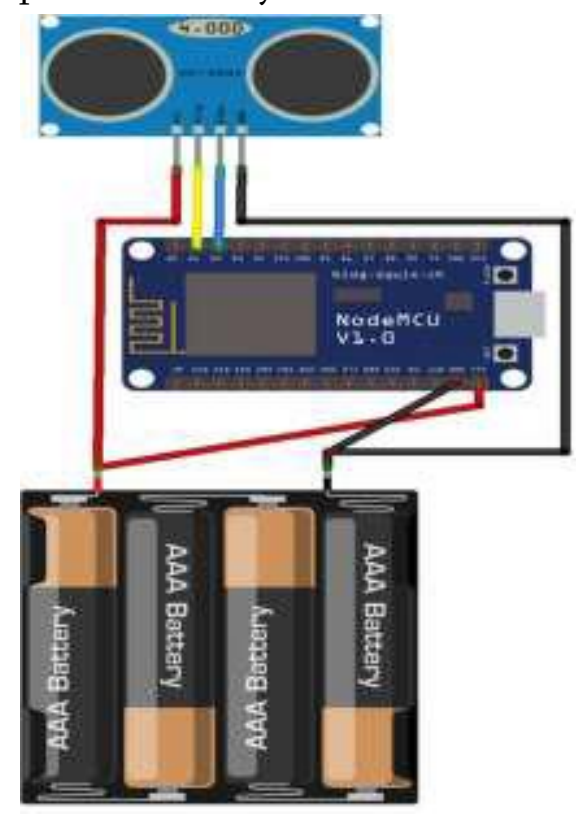

Fig. 5. Design of a microcontroller system in the smart trash.

\section{CONCLUSION}

A practical system to handle the level of trash is being presented in this paper. We can implement real time trash management System by using sensors to check quantity of trash. This system help informing the status of dustbins in real time. Hence the collectors could collect the trash only when the dustbins are full. The ultrasonic sensor range can detect distance in between $2 \mathrm{~cm}$ until $400 \mathrm{~cm}$. Ultrasonic sensor will use the depth of the dustbin to detect the level of trash in the dustbin. However, if there is limited availability of network, the wify module would not work which would be a problem to be considered. A proposed future development of the communication hardware could be done in future. In conclusion any work in the field of reducing the efforts and user intervention has a very bright future.

\section{REFERENCES}

[1]. S. Narang, T. Nalwa, T. Choudhury, and N. Kashyap, "An efficient method for security measurement in internet of things," in International Conference on Communication, Computing and Internet of Things (IC3IoT), 2018.

[2]. L. Celia and Y. Cungang, "(WIP) Authenticated Key Management Protocols for Internet of Things," in IEEE International Congress on Internet of Things (ICIOT, 2018.

[3]. V. A. F. Almeida, D. Doneda, and M. Monteiro, "IEEE International Congress on Internet of Things (ICIOT," Internet Comput., vol. 19, no. 4, pp. 56-59, 2015.

[4]. M. A. Muhal, X. Luo, Z. Mahmood, and A. Ullah, "Physical Unclonable Function Based Authentication Scheme for Smart Devices in Internet of Things," in IEEE International Conference on Smart Internet of Things (SmartIoT), 2018.

[5]. Y. Sharaf-Dabbagh and W. Saad, "Demo Abstract: Cyber-Physical Fingerprinting for Internet of Things Authentication," in IEEE/ACM Second International Conference on Internet-of Things Design and Implementation (IoTDI), 2017.

[6]. Q. Wang and Y. G. Wang, "Research on Power Internet of Things Architecture for Smart Grid Demand," in 2nd IEEE Conference on Energy Internet and Energy System Integration (EI2), 2018.

[7]. S. Singh and N. Singh, "Internet of Things (IoT): Security challenges, business opportunities \& reference architecture for

[8]. Ecommerce," in International Conference on Green Computing and Internet of Things (ICGCIoT), 2015.

[9]. B. Xu, J. Zheng, and Q. Wang, "Analysis and Design of Real-Time Micro-Environment 
Parameter Monitoring System Based on Internet of Things," in International Conference on Internet of

[10]. Things (iThings) and IEEE Green Computing and Communications(GreenCom) and IEEE Cyber, Physical and Social Computing (CPSCom) and IEEE Smart Data (SmartData), 2016.

[11]. Y. Shaikh, V. K. Parvati, and S. R. Biradar, "Survey of Smart Healthcare Systems using Internet of Things (IoT) : (Invited Paper)," in International Conference on Communication, Computing and Internet of Things (IC3IoT), 2018.

[12]. Y. Sun, Y. Xia, H. Song, and R. Bie, "Internet of Things Services for Small Towns," in International Conference on Identification, Information and Knowledge in the Internet of Things, 2014.

\section{Cite this article as :}

Prathikshith Jain, Sowmya K, Ganesh Prasad Navada V, "Smart Trash Bin", International Journal of Scientific Research in Computer Science, Engineering and Information Technology (IJSRCSEIT), ISSN : 2456-3307, Volume 7 Issue 4, pp. 170-175, JulyAugust 2021. Available at doi : https://doi.org/10.32628/CSEIT217429

Journal URL : https://ijsrcseit.com/CSEIT217429 